\title{
Photoinduced modulation and relaxation
}

SUBJECT AREAS:

MATERIALS SCIENCE

OPTICS AND PHOTONICS

Received

11 November 2014

Accepted

3 February 2015

Published

5 March 2015

Correspondence and requests for materials should be addressed to

K.X.J. (jinkx@nwpu. edu.cn) characteristics in $\mathrm{LaAlO}_{3} / \mathrm{SrTiO}_{3}$ heterointerface

\author{
K. X. Jin' ${ }^{1}$, W. Lin' ${ }^{2}$, B. C. Luo' \& T. Wu ${ }^{2}$
}

'Shaanxi Key Laboratory of Condensed Matter Structures and Properties, School of Science, Northwestern Polytechnical University, $X_{i}$ an 710072, China, ${ }^{2}$ Materials Sciences and Engineering, King Abdullah University of Science and Technology, Thuwal 239556900, Saudi Arabia.

We report the modulation and relaxation characteristics in the two-dimensional electron gas system at $\mathrm{LaAlO}_{3} / \mathrm{SrTiO}_{3}$ heterointerface induced by the ultraviolet light illumination $(365 \mathrm{~nm})$. The suppression of Kondo effect at the interface illuminated by the light originates from the light irradiation-induced decoherence effect of localized states. It is interesting to note that the persistent and transient photoinduced effects are simultaneously observed and the photoinduced maximum change values in resistance are $80.8 \%$ and $51.4 \%$ at $T=20 \mathrm{~K}$, respectively. Moreover, the photoinduced relaxation processes after the irradiation are systematically analyzed using the double exponential model. These results provide the deeper understanding of the photoinduced effect and the experimental evidence of tunable Kondo effect in oxides-based two-dimensional electron gas systems.

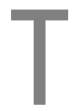

he transition metal oxide heterostructures have attracted considerable attentions in view of finding novel devices in the emergent arena of oxide electronics and microelectronics beyond the conventional semiconductor technology ${ }^{1,2}$. In these epitaxial oxide heterostructures, the (001) heterointerface between $\mathrm{LaAlO}_{3}$ (LAO) and $\mathrm{SrTiO}_{3}(\mathrm{STO})^{3-5}$ is the most fascinating one. A high-mobility two-dimensional electron gas (2DEG) with the order of $3 \times 10^{14} \mathrm{~cm}^{-2}$ is observed at the interface although both materials in bulk form are conventional insulators ${ }^{6-8}$. Subsequently, presences of the $2 D E G$ at $\mathrm{LaGaO}_{3} / \mathrm{STO}^{9}, \mathrm{DyScO}_{3} / \mathrm{STO}^{10}, \mathrm{NdAlO}_{3} / \mathrm{STO}^{11}, \mathrm{GdTiO}_{3} /$ $\mathrm{STO}^{12}$ and $\mathrm{NdGaO}_{3} / \mathrm{STO}$ interfaces ${ }^{13}$, have been experimentally revealed despite the rare $2 D E G$ systems in oxide heterointerfaces. Until now, the heterointerfaces exhibit a wide range of intriguing physical phenomena and properties, such as superconductivity ${ }^{14,15}$, large negative magnetoresistance ${ }^{16}$, ordered magnetic ground states ${ }^{17,18}$, the coexistence of ferromagnetism and superconductivity ${ }^{19-21}$, Kondo effect ${ }^{22,23}$, resistance switching ${ }^{24}$, and so on. Furthermore, the two-dimensional electron gas has the tunability of the conduction state by an atomic force tip ${ }^{25}$, electrostriction ${ }^{26}$, charge and orbital order ${ }^{27}$, and photovolatges ${ }^{28}$, etc. Meanwhile, the photoinduced effect, being the external perturbation of the heterointerface, provides a handy and reliable method to induce the change of properties or the insulator-metal transition ${ }^{29}$. Additionally, photoinduced effects are generally used to photogenerate a hidden electronic phase or the state of matter and unveil the intrinsic physical mechanisms ${ }^{30}$. Some photoinduced phenomena have been discovered in oxides with multiphase coexistence ${ }^{31-33}$. Moreover, the persistent and reversible phases have been observed in the charge-ordering thin film, promising for photonic device applications ${ }^{34}$. Actually, the photoinduced characteristics have been observed in some oxide heterointerfaces with $2 D E G$ systems. For examples, Gennaro et al. and Tebano et al. have studied the persistent photoconductivity at oxide interfaces ${ }^{35,36}$ and Chan et al. have enhanced photoresponse by using Pd nanoparticles ${ }^{37}$. Yamada et al. have deeply analyzed the localization dynamics in the photocarrier recombination process and discussed the interface effects on the electron relaxation dynamics in terms of the strong interface potential ${ }^{38}$. Irvin et al. have investigated the photoresponse on the nanoscale and developed the rewritable oxide photodetectors $^{39}$. Lu et al. have found that the insulating state of the interface can be converted to metallic state by the light illumination and made the LAO/STO interface as a promising nonvolatile memory ${ }^{40}$. Rastogi et al. have investigated the perturbation on photoconducting state by electro static fields ${ }^{41}$. Here, we report the photoinduced phase transition originating from the suppression of Kondo effect and the relaxation process at the LAO/ STO heterointerface with a $10 \mathrm{u}$. c. LAO layer prepared by pulsed laser deposition method ${ }^{42,43}$. Moreover, the persistent and transient photoinduced effects have been systematically investigated. Our results could be helpful 
for further understandings of the photoinduced phenomena in oxide heterostructures and potential applications in the all oxide electronics.

\section{Results and Discussion}

Figure 1 (a) shows the resistance-temperature curves of the heterointerface in darkness. The inset displays the schematic illustration of the heterointerface for the photoinduced measurements. It is observed that the heterointerface exhibits a typical metallic conduction at $T>55 \mathrm{~K}$. When the temperature is lower than $55 \mathrm{~K}$, the resistances are increased as the temperature is decreased. Namely, the sample displays an upturn phenomenon in the resistance of metallic systems at low temperatures, which can be regularly observed in some doped oxide semiconductors ${ }^{44}$ and the $2 D E G$ systems ${ }^{22,23}$. This could be attributed to a Kondo effect ${ }^{45}$, one of the most possible effects in this system, indicating the obvious role of the magnetic interactions among localized and delocalized electrons at low temperatures. Here, the effect might arise from the interplay between the itinerant conduction electrons and localization electrons, which is an admixture composed of localized and unpaired electrons (likely polaronic in nature) ${ }^{46}$. The itinerant conduction electrons are localized at $T<55 \mathrm{~K}$ and as a result the concentration is deceased. The resistance versus temperature curves of the heterointerface in darkness and after the light irradiation at $T<80 \mathrm{~K}$ are presented in Figure 1 (b). It is interesting that the upturn phenomenon in the resistance basically disappears and the curve shows a slightly upward trend after irradiating the heterointerface by the light. The expression of the resistance-temperature curves of the heterointerface in darkness can be described by the expression ${ }^{47}$

$$
R(T)=R_{0}+R_{1} T^{2}+R_{2} T^{5}-R_{3} \ln T
$$

where $R_{0}$ is the residual resistance due to the disorder, the second and the third terms represent the functional temperature dependence of the contributions from electron-electron and electron-photon interactions, respectively. According to Kondo theory, $\ln T$ is the contribution from the exchange coupling between the conducting electrons and the localization electrons. As the solid line in Figure 1(b) shows, the fitting curve agrees with the experimental data well. The obtained fitting parameters are $R_{0}=13487 \Omega, R_{1}=13.3^{*} 10^{-3} \Omega / \mathrm{K}^{2}, R_{2}=$ $7.3^{*} 10^{-7} \Omega / \mathrm{K}^{5}, R_{3}=1348.9 \Omega / \operatorname{lnK}$. After irradiating the sample, the Kondo effect is shrunk. Thereby the total expression of the resistance-temperature relation can be modified as

$$
R(T)=R_{0}+R_{1} T^{2}+R_{2} T^{5}
$$

As shown in Figure 1 (b), the numerical fitting using Eq. (2) to the resistance-temperature curve yields $R_{0}=7265 \Omega, R_{1}=0.19 \Omega / \mathrm{K}^{2}$, and $R_{2}=8.7^{*} 10^{-6} \Omega / \mathrm{K}^{5}$. The Kondo effect at low temperature is suppressed by the photocarriers injection, similar to effects of the dopant and magnetic field ${ }^{48,49}$. The suppression effect might originate from the light irradiation-induced decoherence effect of localized states. At the LAO/STO interface, the orbital reconstruction due to the broken symmetry is well established. Particularly, the degeneracy of the Ti $t_{2 g}$ state is lifted at the interface, and the $\mathrm{Ti} 3 \mathrm{~d}_{\mathrm{xy}}$ state possesses the lowest energy. Accordingly, the $3 \mathrm{~d}_{\mathrm{xy}}$-derived band is occupied firstly and the electrons are highly susceptible to the localization. When the light irradiates the heterointerface, the photocarriers induce the delocalization of electrons. Thus, the Kondo effect is suppressed and the photoinduced phase transition from the insulating to metallic state emerges at low temperatures. Similar results were found in a quantum dot induced by the external irradiation ${ }^{50}$.

As shown in Figure 1 (c), the resistances of the heterointerface are decreased from the $8.7 \mathrm{k} \Omega$ to $4.3 \mathrm{k} \Omega$ at $T=20 \mathrm{~K}$ when it is irradiated by the light. It is clear that the resistance is restored to a steady value and then maintains this value for the long time when the light is off. Namely, the heterointerface favors the persistent photoinduced
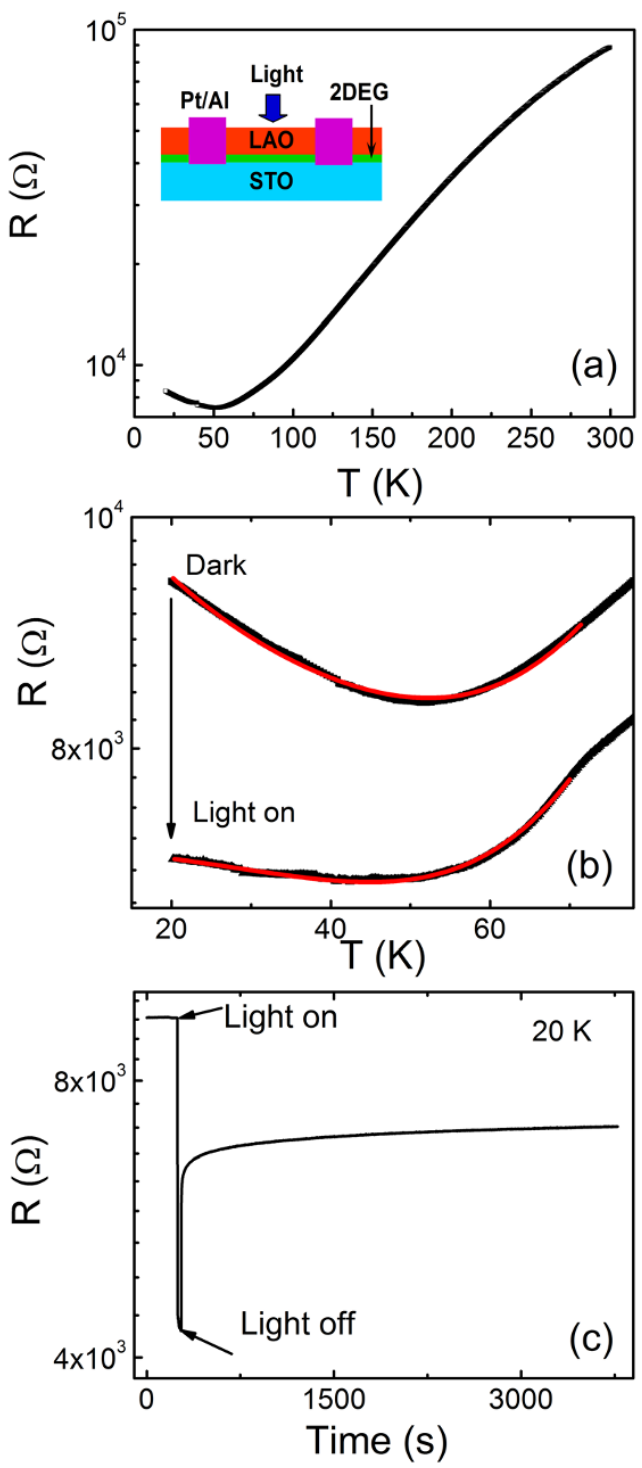

Figure 1 (a) Resistance-temperature curves of the heterointerface in darkness, and the inset displays the schematic illustration of the heterointerface for the photoinduced measurement. (b) Resistance versus temperature curves of the heterointerface with and without the light irradiation at $T<80 \mathrm{~K}$. The solid lines are the fitted curves. (c) Time dependence of the resistance in the heterointerface at $20 \mathrm{~K}$.

effect. The conduction mechanisms of the $2 D E G$ system have three possible explanations: the electronic reconstruction caused by the polar catastrophe, the La interdiffusion through the interface, and the defect generation (such as oxygen vacancies) in the STO substrate $^{51}$. Here, we account for the photoinduced effect based on the electronic reconstruction scenario, which is a displacement of electrons from the outer region of $\mathrm{LAO}$ into the $\mathrm{Ti} 3 \mathrm{~d}$ states of the topmost STO layers. And this is driven by the relaxation of the electrostatic energy accumulated in the polar layer, forming the built-in field. Thus, the polar layer plays an important role in the persistent photoinduced effect. Besides, the oxygen vacancies generated during the preparation of the film are inevitable and thus affect the photoindueced effect by the formation of the subbands. Likewise, the persistent photoconductivity is also observed in some semiconductor-based interfaces with a $2 D E G$ system, which is explained in terms of a separation of the electron-hole pairs by the built-electric field ${ }^{52-54}$. The separation results in the long lifetime of recombination process. The photon energy $(\sim 3.3 \mathrm{eV})$ of the light with the wave- 
length of $365 \mathrm{~nm}$ is smaller than the band gap of LAO $(\sim 5.6 \mathrm{eV})$ and larger than that of STO $(\sim 3.2 \mathrm{eV})^{55}$. Thereby the light can irradiate the $2 D E G$ interface at STO side and generate the electron-hole pairs by passing through the LAO layer, resulting in an increase in the $2 D E G$ density. The excited electrons falling in the triangular interfacial potential well in STO side are added to $2 D E G$ systems and holes are localized by shallow defects. When the light is off, the trapped electrons in the potential well will not recombine with holes due to the energy barrier. Thereby the persistent photoinduced effect occurs. The decay dynamics of photoinduced effects are determined by the lifetime of the recombination probability of the photoinduced electron-hole pairs. Actually, the STO single crystals also exhibit the persistent photoconductivity when exposed to subband gap light, which is attributed to the excitation of an electron from a titanium vacancy defect into the conduction band ${ }^{56}$. In this paper, the photon energy with the value of about $3.3 \mathrm{eV}$ is slightly higher than the band gap of STO. Moreover, we have only found the transient photoinduced effect and an insulator-metal phase transition in a bare STO single crystal at the same condition ${ }^{31}$. This further confirms the effect of polar discontinuity at the interface.

In order to further investigate the photoinduced response process, we performed the experiments on the time dependence of resistances. As shown in Figure 2, the time dependence of the resistance at different temperatures, (a) $20 \mathrm{~K}$, (b) $80 \mathrm{~K}$, (c) $160 \mathrm{~K}$, and (d) $300 \mathrm{~K}$ is displayed. The resistances quickly decrease to the minimum values when the light is on, and then show a decay to a steady value when the light is off. It is worthwhile to note that the heterointerface under the first irradiation shows the persistent photoinduced effect and then restores to a balanced state. After that, the heterointerface exhibits the transient photoinduced effect and has the excellent repetition when the heterointerface is irradiated again. Here, the persistent photoinduced change in the resistance $(P R)$ is defined as $\left(R_{0}-R_{\mathrm{p}}\right) /$ $R_{\mathrm{p}}$, where $R_{\mathrm{p}}$ is the resistance of the sample irradiated by the light, and $R_{0}$ is the original resistance without the light irradiation. Meanwhile, the transient photoindcued change in the resistance (TR) is defined as $\left(R_{\mathrm{b}}-R_{\mathrm{p}}\right) / R_{\mathrm{p}}$, where $R_{\mathrm{b}}$ is the resistance of the balanced state after the first irradiation. The $P R$ and $T R$ dependences of temperature are shown in Figure 3. As we can see, the $P R$ and $T R$ effects strongly depend on the temperature. Both the values of the $P R$ and $T R$ show the same tendency and decease with increasing the temperature, which is ascribed to the thermal fluctuations at higher temperatures $^{57}$. The maximum values of the $P R$ and the $T R$ are $80.8 \%$ and $51.4 \%$ at $T=20 \mathrm{~K}$, respectively. Considering both the band bending in the polar layer as mentioned by the electronic reconstruction scenario ${ }^{58,59}$ and subbands in STO, the band diagram is displayed in the inset of Figure 3, providing a sketch of states at the interfaces. We can see that direct promotion from the valence band maximum (VBM) to the conduction band minimum $(C B M)$ is made under the irradiation of $365 \mathrm{~nm}$ light (process (1)). Then the photoexcited electrons drift to the channel region (process (2)), increasing the $2 D E G$ density and then contributing to the observed decrease in the resistance. When the light illumination is off, the photoexcited electrons will be recombinated with holes at the VBM in STO (process (3)) and be recaptured slowly by the subbands (process (4) in the figure. Taking account into results above, we speculate that the photoinduced effect might contain two parts. One is the process that is not recovered, originating from the effect of polar layer at the $2 D E G$ interface (process (2)). The other is the process, which can
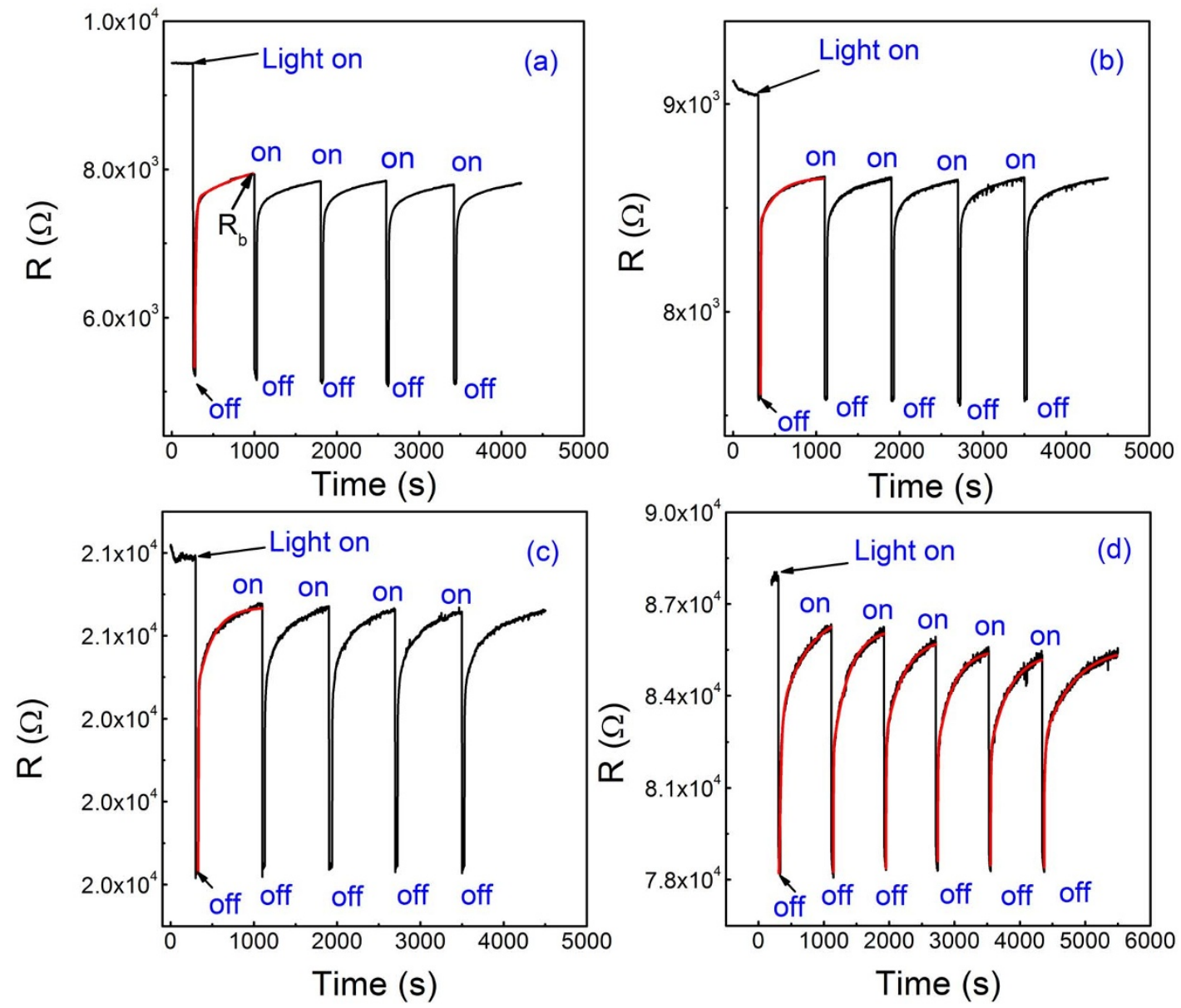

Figure $2 \mid$ Time dependence of the resistance at different temperatures, (a) $20 \mathrm{~K}$, (b) $80 \mathrm{~K}$, (c) $160 \mathrm{~K}$, (d) $300 \mathrm{~K}$. The red solid lines are the fitting curves. 


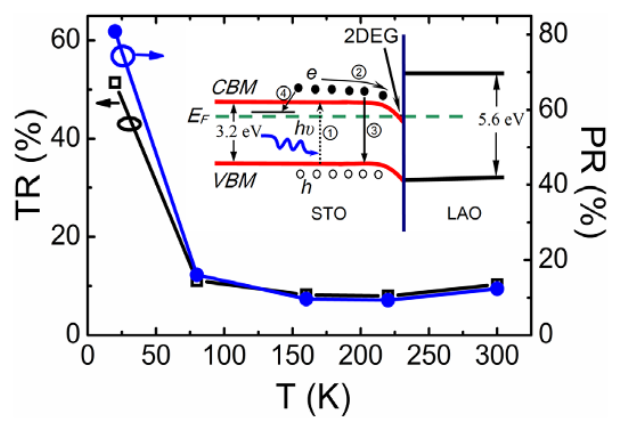

Figure 3 Persistent and transient photoinduced change in the resistance of heterointerface as a function of temperature. Inset: schematic diagram of band structure at the polar/non-polar interface where the band bending is displayed.

be recovered and related with the intrinsic STO. This includes the recombination of electrons in the conduction band and holes in the valence band of STO (process (3) and recapture of electrons into the subbands (process (4)) after the irradiation.

To obtain insight into the persistent and transient photoinduced effect, we further analyzed the relaxation characteristics of the process when the light is off. The resistances increase nonlinearly with the irradiation time when the light is off at $T=20 \mathrm{~K}, 80 \mathrm{~K}, 160 \mathrm{~K}$ and $300 \mathrm{~K}$ as shown in Figure 2. The recovery of the resistance after the irradiation is the relaxation process of carriers, indicating the carrier's dynamics of recombination. The single exponential function can't describe the decay process very well and thus we make a fit using the double exponential function due to the two fitting parameters, which dominate the fast and slow processes, respectively. As shown in Figure 2, the resistance vs. time curves of STO after the irradiation can be fitted by the following formula:

$$
R=A \exp \left(t / \tau_{1}\right)+B \exp \left(t / \tau_{2}\right)+C
$$

where $A$ and $B$ are the magnitude, $t$ is the time, $\tau_{1}$ and $\tau_{2}$ is the fast and slow time constant of relaxation process, respectively. The time impendent term $C$ is presumably due to the heating effect lasting for longer time than time range concerned here. Usually the bandband excitation is very fast and related with the initial excitation of electrons to higher energy states. A longer generation lifetime is mostly caused by a more complex process, involving the trapping and thermal activation processes. Hence, that exhibits probability for a lattice relaxation at the surface, in which shallow energy levels in the band gap form when shallow donors convert into deep donors. Such separation prevents the recombination process and leads to very long lifetimes of the photoexcited recovery. The exponential model pro- vides an improved description of the photoinduced decay process. The red lines represent the fitting curves and a good agreement between the fitted and experimental data is obtained as shown in Figure 2. We can get the values of the time constant at different temperatures from the fitting. Figure 4 (a) shows the time constants of the heterointerface when the light is off as a function of temperature. It is observed that both the fast and slow time constants decrease to a minimum value at about $160 \mathrm{~K}$ firstly, and then are followed by an increase. The time constants are the characteristics of relaxation process in the photoinduced resistance change, and there exists the similar phenomena in the magnetization and the spin glasses of the manganites ${ }^{60,61}$. It is important to note that the temperature corresponding to the minimum value is roughly close to that of the cubic-to-tetragonal structural phase transition of STO $(\sim 105 \mathrm{~K})$. So, we believe that such a phase transition is significant due to its latent paraelectric nature, which is intimately associated with the $d^{0}$-ness of the Ti ions in STO because the light can irradiate the STO through the LAO layer. Additionally, the occupation of Ti with extra electrons being doped at interface is modified due to the polar layers. Therefore the interface also plays an important role although we expect the same to happen deep down in the substrate ${ }^{62}$. Meanwhile, the time decay is dependent on temperature. This behavior can be explained as the probability of the thermal activation of the localized carriers to overcome the potential barrier. Then the thermal carriers escape to the recombination channels. Thus, we express the temperature dependence of the slow time constant by following the Arrhenius-law ${ }^{63}$, which is expressed by: $\tau_{2}=\tau_{0} e^{-E / k T}$, where $\tau_{0}$ is the high temperature limit of time constant, $E$ is the thermally activated energy for the thermal capture of an electron at deep levels, and $k$ is Boltzmann constant. Figure 4 (b) shows Arrhenius plot of $\tau_{2}$ with temperature. The red solid lines are the linear fitting curves and the time constants agree with the formula. Clearly, it is observed that two distinct temperature regions of activation process exist in the Arrhenius plot at about $160 \mathrm{~K}$, which is consistent with the temperature dependence of the time constant. From the fitting, the calculated value of $E$ is about $0.04 \mathrm{meV}$ at low temperatures $T<160 \mathrm{~K}$. However, at higher temperatures, the $E$ is estimated as $1.12 \mathrm{meV}$, which is almost three times higher in comparison to the activation energy at low temperatures. The obtained values are approximately consistent with the previously reported values in oxides and GaN based $2 D E G$ systems ${ }^{62,64}$. Nevertheless, the obtained values are smaller than that. The two-dimensional electron gas is thought to be formed from the three $3 d-t_{2 g}$ states. The energy scale for the electric subband states is expected to be of the order of tenths of an $\mathrm{eV}$, determined by an interface electric field, electron density, and strain ${ }^{65}$. The energy scale in our experiments is on the scale of $\mathrm{meV}$, which is attributed to the different depths of the localization at subbands of STO. The smaller activation energy
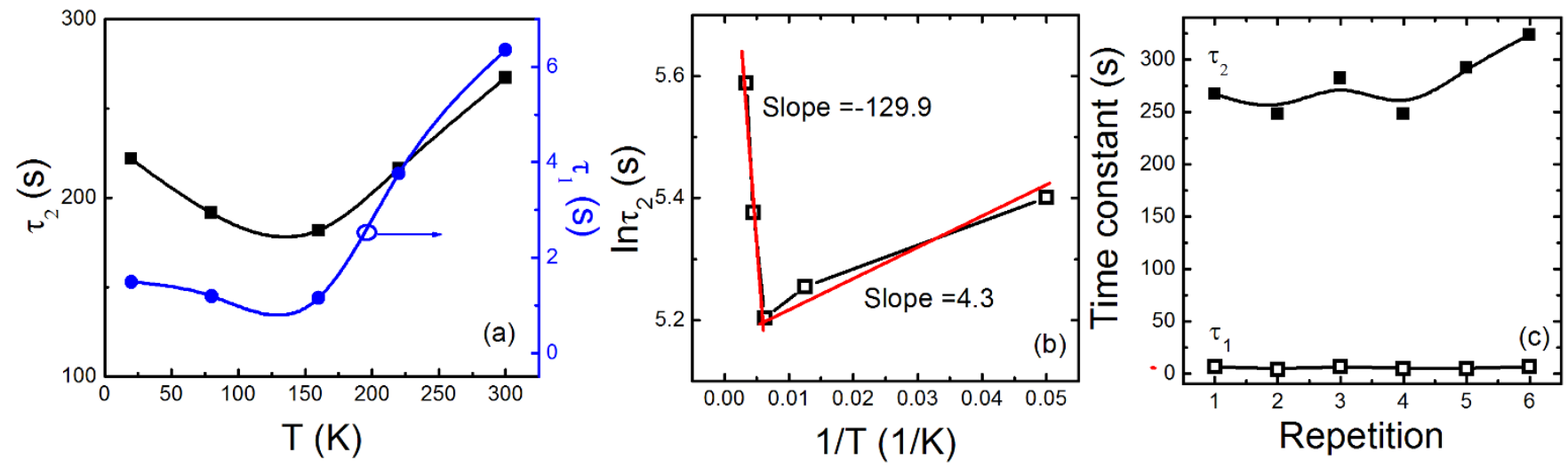

Figure $4 \mid$ (a) Time constants of the heterointerface when the light is off as a function of temperature. (b) Logarithm of the time constant $\left(\tau_{2}\right)$ as a function of reciprocal temperature. The red solid lines are the fitting curves. (c) Time constants dependence of repetition times. 
means that the depth of localizations is shallower for our heterointerface. Considering the practical applications, we further analyze the characteristics of repetition at $300 \mathrm{~K}$. Figure 4 (c) shows the time constants obtained from the fitting done using Figure 2 (d) dependence of repetition times. Both the fast and slow time constants slightly increase with increasing the repetition times, indicating the transient photoinduced effect has nice repetitions. We expect that the investigation following the results can enhance our understanding of the electrical and optical properties in $2 D E G$ systems. In summary, we investigate the photoinduced modulation and relaxation characteristics in the two-dimensional electron gas system at LAO/STO heterointerface irradiated by the ultraviolet light $(365 \mathrm{~nm})$. The Kondo effect at the interface is suppressed by the light, which originates from the light irradiation-induced decoherence effect of localized states. The persistent and transient photoinduced effects are simultaneously observed and the minimum values of time constants gained from the double exponential model appear. These results would open the way for studying very fundamental solid state phenomena confined to an interface and suggest potential applications in the development of optical detectors using oxides-based devices.

\section{Methods}

The 10 u. c. $\mathrm{LAO}$ film was grown on $\mathrm{TiO}_{2}$-terminated $\mathrm{STO}$ substrates at $800^{\circ} \mathrm{C}$ under an oxygen pressure of $10^{-3} \mathrm{mbar}$ using the pulsed laser deposition method. The repetition rate of the $\mathrm{KrF}$ laser is $1 \mathrm{~Hz}$ and the fluence is $1.5 \mathrm{~J} / \mathrm{cm}^{2}$. The film growth was monitored by in situ high-pressure reflection high-energy electron diffraction (RHEED). The RHEED intensity oscillation indicates a layer-by-layer growth mode. In order to obtain an Ohmic contact, the $\mathrm{Pt} / \mathrm{Al}$ square-shaped electrodes with the size of $0.5 \mathrm{~mm} * 0.5 \mathrm{~mm}$ were used as electrodes. The distance between two electrodes is about $0.5 \mathrm{~mm}$. The sample was placed in a Janis CCS-300 closed-circuit refrigerator cryostat with quartz glass windows. The Keithley multimeter (model 2635A) was used for the resistance-temperature measurements in the temperature range from 20 to $300 \mathrm{~K}$. The ultraviolet light with the wavelength of $365 \mathrm{~nm}$ and the power density of $2.6 \mathrm{~W} / \mathrm{cm}^{2}$ typically illuminated the sample. Before the light illumination, the samples were kept in darkness for $10 \mathrm{~h}$ for the data measured until the resistance was stabilized.

1. Zubko, P., Gariglio, S., Gabay, M., Ghose, P. \& Triscone, J. M. Interface physics in complex oxide heterostructures. Annu. Rev. Cond. Matt. Phys. 2, 141-165(2011).

2. Cen, C., Thiel, S., Mannhart, J. \& Levy, J. Oxide Nanoelectronics on Demand. Science 323, 1026-1030(2009).

3. Ohtomo, A. \& Hwang, H. Y. A high-mobility electron gas at the $\mathrm{LaAlO}_{3} / \mathrm{SrTiO}_{3}$ heterointerface. Nature 427, 423-426(2004).

4. Mannhart, J. \& Schlom, D. G. Oxide interfaces-an opportunity for electronics. Science 327, 1607-1611(2010).

5. Jang, H. W. et al. Metallic and insulating oxide interfaces controlled by electronic correlations. Science 331, 886-889 (2011).

6. Moetakef, P. et al. Electrostatic carrier doping of $\mathrm{GdTiO}_{3} / \mathrm{SrTiO}_{3}$ interfaces. Appl. Phys. Lett. 99, 232116(2011).

7. Caviglia, A. D. et al. Electric field control of the $\mathrm{LaAlO}_{3} / \mathrm{SrTiO}_{3}$ interface ground state. Nature 456, 624-627(2008).

8. Au, K., Li, D. F., Chan, N. Y. \& Dai, J. Y. Polar liquid molecule induced transport property modulation at $\mathrm{LaAlO}_{3} / \mathrm{SrTiO}_{3}$ heterointerface. Adv. Mater. 24, 2598-2602(2012).

9. Perna, P. et al. Conducting interfaces between band insulating oxides: the $\mathrm{LaGaO}_{3} / \mathrm{SrTiO}_{3}$ heterostructure. Appl. Phys. Lett. 97, 152111(2010).

10. Li, D. F., Wang, Y. \& Dai, J. Y. Tunable electronic transport properties of $\mathrm{DyScO}_{3} /$ $\mathrm{SrTiO}_{3}$ polar heterointerface. Appl. Phys. Lett. 98, 122108(2011).

11. Annadi, A. et al. Evolution of variable range hopping in strongly localized two dimensional electron gas at $\mathrm{NdAlO}_{3} / \mathrm{SrTiO}_{3}(100)$ heterointerfaces. Appl. Phys Lett. 101, 231604(2012).

12. Moetakef, P. et al. Transport in ferromagnetic $\mathrm{GdTiO}_{3} / \mathrm{SrTiO}_{3}$ heterostructures. Appl. Phys. Lett. 98, 112110(2011).

13. Gunkel, F. et al. Stoichiometry dependence and thermal stability of conducting $\mathrm{NdGaO}_{3} / \mathrm{SrTiO}_{3}$ heterointerfaces. Appl. Phys. Lett. 102, 071601(2013).

14. Reyren, N. et al. Superconducting interfaces between insulating oxides. Science 317, 1196-1199(2007).

15. Ueno, K. et al. Electric-field-induced superconductivity in an insulator. Nat. Mater. 7, 855-858(2008)

16. Brinkman, A. et al. Magnetic effects at the interface between non-magnetic oxides. Nat. Mater. 6, 493-496(2007).

17. Moetakef, P. et al. Carrier-controlled ferromagnetism in $\mathrm{SrTiO}_{3}$. Phys. Rev. X 2 , 021014(2012).

18. Michaeli, K., Potter, A. C. \& Lee, P. A. Superconducting and ferromagnetic phases in $\mathrm{SrTiO}_{3} / \mathrm{LaAlO}_{3}$ oxide interface structures: possibility of finite momentum pairing. Phys. Rev. Lett. 108, 117003(2012).
19. Bert, J. A. et al. Direct imaging of the coexistence of ferromagnetism and superconductivity at the $\mathrm{LaAlO}_{3} / \mathrm{SrTiO}_{3}$ interface. Nat. Phys. 7, 767-771(2011)

20. Dikin, D. A. et al. Coexistence of superconductivity and ferromagnetism in two dimensions. Phys. Rev. Lett. 107, 056802(2011).

21. Li, L., Richter, C. Manhart, J \& Ashoori, R. C. Coexistence of magnetic order and two-dimensional superconductivity at $\mathrm{LaAlO}_{3} / \mathrm{SrTiO}_{3}$ interfaces. Nat. Phys. 7 , 762-766(2011).

22. Wang, X. et al. Magnetoresistance of two-dimensional and three-dimensional electron gas in $\mathrm{LaAlO}_{3} / \mathrm{SrTiO}_{3}$ heterostructures: influence of magnetic ordering, interface scattering, and dimensionality. Phys. Rev. B 84, 075312(2011).

23. Ruhman, J., Joshua, A., IIani, S. \& Altman E. Competition between Kondo screening and magnetism at the $\mathrm{LaAlO}_{3} / \mathrm{SrTiO}_{3}$ interface. Phys. Rev. B 90, 125123(2014)

24. Wu, S. X. et al. Bipolar resistance switching in transparent ITO/LaAlO $/ \mathrm{SrTiO}_{3}$ memristors. ACS Appl. Mater. Interfaces 6, 8575-8579(2014).

25. Cen, C. et al. Nanoscale control of an interfacial metal insulator transition at room temperature. Nat. Mater. 7, 298-302(2008).

26. Cancellieri, C. et al. Electrostriction at the $\mathrm{LaAlO}_{3} / \mathrm{SrTiO}_{3}$ interface. Phys. Rev. Lett. 107, 056102(2011).

27. Rubano, A. et al. Spectral and spatial distribution of polarization at the $\mathrm{LaAlO}_{3} /$ $\mathrm{SrTiO}_{3}$ interface. Phys. Rev. B 83, 155405(2011).

28. Liang, H. X. et al. Giant photovoltaic effects driven by residual polar field within unit-cell-scale $\mathrm{LaAlO}_{3}$ films on $\mathrm{SrTiO}_{3}$. Sci. Rep. 3, 1975(2013).

29. Ju, L. et al. Photoinduced doping in heterostructures of graphene and boron nitride. Nat. Nano. 9, 348-352(2014).

30. Tokura, Y. Photoinduced phase transition: a tool for generating a hidden state of matter. J. Phys. Soc. Jpn. 75, 011001-011008(2006).

31. Jin, K. X., Luo, B. C., Li, Y. F., Chen, C. L. \& Wu, T. Photoinduced phase transition and relaxation in bare $\mathrm{SrTiO}_{3}$ single crystals. J. Appl. Phys. 114, 033509(2013).

32. Fiebig, M., Miyano, K., Tomioka, Y. \& Tokura, Y. Visualization of the local insulator-metal transition in $\mathrm{Pr}_{0.7} \mathrm{Ca}_{0.3} \mathrm{MnO}_{3}$. Science 280, 1925-1928(1998).

33. Smolyaninov, I. I. et al. High resolution study of permanent photoinduced reflectivity changes and charge-order domain switching in $\mathrm{Bi}_{0.3} \mathrm{Ca}_{0.7} \mathrm{MnO}_{3}$. Phys. Rev. Lett. 87, 127204(2001).

34. Takubo, N. et al. Persistent and reversible all-optical phase control in a manganite thin film. Phys. Rev. Lett. 95, 017404(2005).

35. Gennaro, E. D. et al. Persistent photoconductivity in $2 \mathrm{D}$ electron gases at different oxide interfaces. Adv. Opt. Mater. 1, 834-843(2013).

36. Tebano, A., Fabbri, E., Pergolesi, D., Balestrino, G. \& Traversa, E. Roomtemperature giant persistent photoconductivity in $\mathrm{SrTiO}_{3} / \mathrm{LaAlO}_{3}$ heterostructures. ACS Nano 6, 1278-1283 (2012).

37. Chan, N. Y. et al. Palladium nanoparticle enhanced giant photoresponse at $\mathrm{LaAlO}_{3} / \mathrm{SrTiO}_{3}$ two-dimensional electron gas heterostructures. ACS Nano 7 , 8673-8679(2013).

38. Yamada, Y., Sato, H. K., Hikita, Y., Hwang, H. Y. \& Kanemitsu, Y. Photocarrie recombination and localization dynamics of $\mathrm{LaAlO}_{3} / \mathrm{SrTiO}_{3}$ heterostructures. Pro. SPIE 8987, 898710(2014).

39. Irvin, P. et al. Rewritable nanoscale oxide photodetector. Nat. Photon. 4, 849-852(2010).

40. Lu, H. L. et al. Reversible insulator-metal transition of $\mathrm{LaAlO}_{3} / \mathrm{SrTiO}_{3}$ interface for nonvolatile memory. Sci. Rep. 3, 2870(2013).

41. Rastogi, A. et al. Photoconducting state and its perturbation by electro static fields in oxide-based two-dimensional electron gas. Phys. Rev. B 86, 075127(2012).

42. Lin, W. N. et al. Electrostatic modulation of $\mathrm{LaAlO}_{3} / \mathrm{SrTiO}_{3}$ interface transport in an electric double-layer transistor. Adv. Mater. Interfaces 1, 1300001(2014).

43. Wu, S. X. et al. Nonvolatile resistive switching in $\mathrm{Pt} / \mathrm{LaAlO}_{3} / \mathrm{SrTiO}_{3}$ heterostructures. Phys. Rev. X 3, 041027(2013)

44. Li, Y. F. et al. Electrostatic tuning of Kondo effect in a rare-earth-doped wideband-gap oxide. Phys. Rev. B 87, 155151(2013).

45. Kondo, J. Resistance minimum in dilute magnetic alloys. Prog. Theor. Phys. 32 37-49 (1964)

46. Lee, M., Williams, J. R., Zhang, S. P., Frisbie, C. D. \& Goldhaber-Gordon, D. Electrolyte gate-controlled Kondo effect in $\mathrm{SrTiO}_{3}$. Phys. Rev. Lett. 107, 256601(2011)

47. Banerjee, N., Huijben, M., Koster, G. \& Rijnders, G. Direct patterning of functional interfaces in oxide heterostructures, Appl. Phys. Lett. 100, 041601(2012)

48. Lin, C. P., Hsu, C. Y., Sun, S. J. \& Chou, H. The Kondo effect and carrier transport in amorphous Cr-doped $\mathrm{In}_{2} \mathrm{O}_{3}$ thin films. AIP Advances 2, 042186(2012).

49. Li, M. Y., Graf, T., Schladt, T. D., Jiang, X. \& Parkin, S. S. P. Role of percolation in the conductance of electrolyte-gated $\mathrm{SrTiO}_{3}$. Phys. Rev. Lett. 109, 196803(2012).

50. Kaminski, A., Nazarov, Y. V. \& Glazman, L. I. Suppression of the Kondo effect in a quantum dot by external irradiation. Phys. Rev. Lett. 83, 384(1999).

51. Lee, S. W., Liu, Y., Heo, J., \& Gordon, R. G. Creation and control of twodimensional electron gas using $\mathrm{Al}$-based amorphous oxides/ $/ \mathrm{SrTiO}_{3}$ heterostructures grown by atomic layer deposition. Nano Lett.12 4775-4783(2012).

52. Li, B. K., Ge, W. K., Wang, J. N. \& Chen, K. J. Persistent photoconductivity and carrier transport in $\mathrm{AlGaN} / \mathrm{GaN}$ heterostructures treated by fluorine plasma. Appl. Phys. Lett. 92, 082105(2008). 
53. Li, J. Z., Lin, J. Y., Jiang, H. X., Khan, M. A. \& Chen, Q. Persistent photoconductivity in a two-dimensional electron gas system formed by an AlGaN/GaN heterostructure. J. Appl. Phys. 82, 1227(1997).

54. Kioupakis, E., Yan, Q. M. \& Van de Walle, C. G. Interplay of polarization fields and Auger recombination in the efficiency droop of nitride light-emitting diodes. Appl. Phys. Lett. 101, 231107(2012).

55. Jin, K. X. et al. Self-powered ultraviolet photovoltaic effects based on metal/ $/ \mathrm{SrTiO}_{3}$ Schottky junctions. Europhysics Letters 103, 57007(2013).

56. Tarun, M. C., Selim, F. A. \& McCluskey, M. D. Persistent photoconductivity in strontium titanate, Phys. Rev. Lett. 111, 187403(2013).

57. Jin, K. X., Zhao, S. G., Tan, X. Y., Chen, C. L. \& Jia, X. W. Transport and photoinduced properties in highly Sr-deficient manganite films. Appl. Phys. A 95, 789-792(2009).

58. Stengel, M. First-principles modeling of electrostatically doped perovskite systems. Phys. Rev. Lett. 106, 136803(2011)

59. Singh-Bhalla, G. et al. Built-in and induced polarization across $\mathrm{LaAlO}_{3} / \mathrm{SrTiO}_{3}$ heterojunctions. Nat. Phys. 7, 80-86(2011).

60. Coey, J. M. D., Ryan, D. H. \& Buder, R. Kohlrausch thermal relaxation in a random magnet. Phys. Rev. Lett. 58, 385-388(1987).

61. Zhao, S. G., Jin, K. X. \& Chen, C. L. Photoinduced effect in charge-ordering $\mathrm{La}_{0.5} \mathrm{Ca}_{0.5} \mathrm{MnO}_{3}$ film. J. Appl. Phys. 101, 083701(2007).

62. Rastogi, A., Pulikkotil, J. J. \& Budhani, R. C. Enhanced persistent photoconductivity in $\delta$-doped $\mathrm{LaAlO}_{3} / \mathrm{SrTiO}_{3}$ heterostructures. Phys. Rev. B 89, 125127(2014).

63. Liu, C. et al. Colossal dielectric constant and relaxation behaviors in $\mathrm{Pr}_{\mathrm{SrTiO}}$ ceramics. J. Appl. Phys. 107, 094108(2010).

64. Lin, T. Y. et al. Two-dimensional electron gas and persistent photoconductivity in $\mathrm{Al}_{\mathrm{x}} \mathrm{Ga}_{1-\mathrm{x}} \mathrm{N} / \mathrm{GaN}$ heterostructures. Phys. Rev. B 58, 13793-13798(1998).
65. Allen, S. J. et al. Conduction-band edge and Shubnikov-de Haas effect in low electron density $\mathrm{SrTiO}_{3}$. Phys. Rev. B 88, 045114(2013).

\section{Acknowledgments}

This work is supported by the National Natural Science Foundation of China (Nos. 61078057, 51202195 and 51172183) and NPU Foundation for Fundamental Research (No. JC20120246).

\section{Author contributions}

K.X. and T.W. conceived and designed the experiments. W.L. prepared the sample. K.X. And B.C. performed the experiments. All authors discussed the results and conmented on the manuscript.

\section{Additional information}

Competing financial interests: The authors declare no competing financial interests. How to cite this article: Jin, K.X., Lin, W., Luo, B.C. \& Wu, T. Photoinduced modulation and relaxation characteristics in $\mathrm{LaAlO}_{3} / \mathrm{SrTiO}_{3}$ heterointerface. Sci. Rep. 5, 8778; DOI:10.1038/srep08778 (2015).

(C) This work is licensed under a Creative Commons Attribution 4.0 Internationa License. The images or other third party material in this article are included in the article's Creative Commons license, unless indicated otherwise in the credit line; if the material is not included under the Creative Commons license, users will need to obtain permission from the license holder in order to reproduce the material. To view a copy of this license, visit http://creativecommons.org/licenses/by/4.0/ 